\title{
Diffuse hepatocellular carcinoma with extension into the right atrium
}

\begin{abstract}
Hepatocellular carcinoma is one of the diagnoses that we most frequently see in our clinical practice, accustomed to seeing it as a single lesion, many do not know the diffuse subtype whose involvement and extension are rare. In this article, we present a case of diffuse hepatocarcinoma with cardiac extension and we discuss the importance of recognizing it because its management and prognosis depend on it.
\end{abstract}

Keywords: carcinoma hepatocelular, liver neoplasms, magnetic resonance imaging
Volume 12 Issue 6 - 2021

\author{
Jennifer Richardson,' Saray Serrano, ${ }^{2}$ Marco \\ Luján ${ }^{3}$ \\ 'Radiology Resident, Faculty of Medicine, Universidad del Norte, \\ Colombia \\ ${ }^{2}$ Medical Radiologist, Cediul S.A., Colombia \\ Medical Hepatologist, Clínica de la Costa, Colombia
}

Correspondence: Jennifer Richardson, Radiology Resident, Faculty of Medicine, Universidad del Norte, BarranquillaColombia,Email jrichardson@uninorte.edu.co

Received: November 09, 202I | Published: December 06 , 202

\section{Introduction}

Primary hepatic carcinoma represents the second leading cause of cancer mortality and is the fifth most prevalent tumor, ${ }^{1}$ with hepatocellular carcinoma (HCC) as the most common histological type accounting for $90 \%$ of these lesions. ${ }^{2}$ It is a multicausal pathology secondary to genetic changes favored by chronic liver disease, cirrhosis, hepatitis $\mathrm{B}$ and $\mathrm{C}$ virus infection, excessive alcohol consumption, obesity, metabolic syndrome, non-alcoholic hepatic steatosis, smoking, hemochromatosis, type 2 diabetes mellitus, Wilson's disease, congenital biliary atresia, and aflatoxins. The coexistence of these diseases increases the incidence of the tumor. $^{3}$ According to its growth pattern, it is classified into: nodular (multifocal), massive (focal), and infiltrative (diffuse). ${ }^{4}$

\section{Clinical scenario}

A 48-year-old male patient, with a history of liver cirrhosis due to hepatitis B and alcoholism, consults for an increase in the abdominal perimeter that hinders food intake. To the physical examination with abdominal distension. In blood biochemistry, alpha-fetal high protein of $500 \mathrm{ng} / \mathrm{ml}$ stands out. In the cardiac and simple abdominal magnetic resonance with contrast, in which a cirrhotic liver with a hypervascular lesion, irregular heterogeneous lesion in segment VIII, is observed, it measures approximately $6 \times 4.8 \times 3.7 \mathrm{cms}$ (Figure 1A). It presents late-stage washing with capsular enhancement (Figure 1B). In addition, the lesion invades the portal vein with tumor thrombus (Figure 2), and inferior cava extending to the right atrium (Figure 3), where it occupies more than $50 \%$ of it with valvular contact (Figure 4). These findings are related to diffuse hepatocellular carcinoma with metastatic involvement.

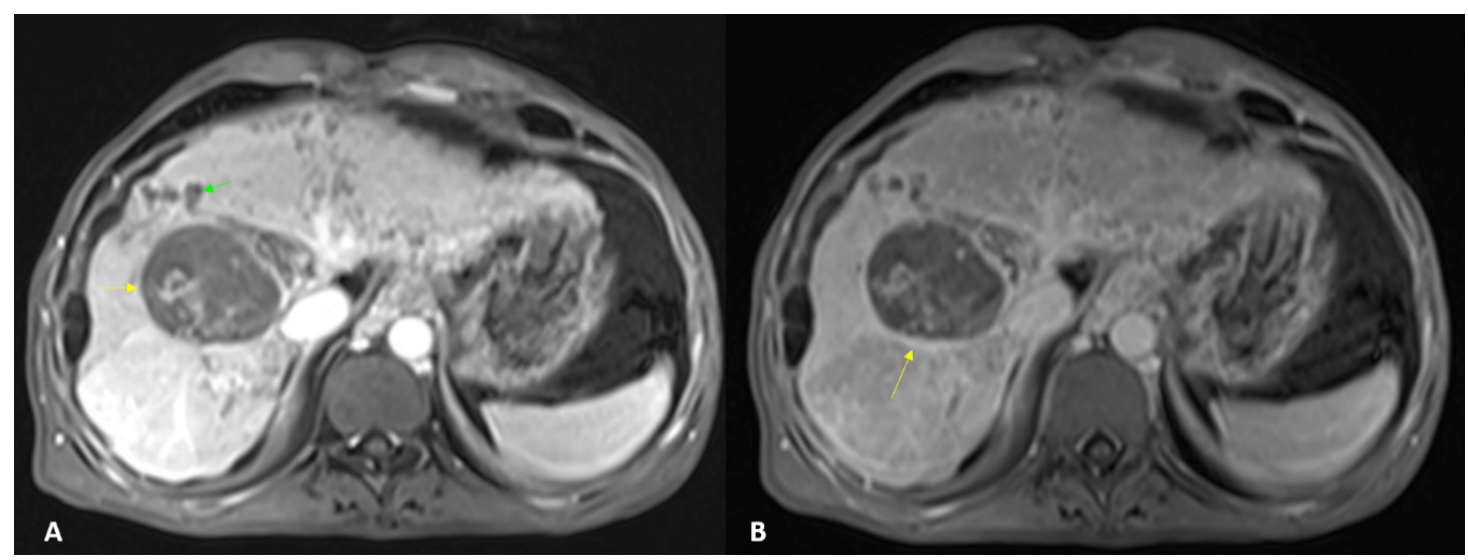

Figure I Description: Axial magnetic resonance imaging in TIW. Hepatocarcinoma in segment VIII with serpentiginous enhancement inside in relation to intratumor neoformation (A), and enhancement of its capsule in late phases (B). Note the dilation of the intrahepatic bile duct (green arrow).

Origin: Department of Radiology, Clínica de la Costa, Barranquilla, Colombia, 2019. 


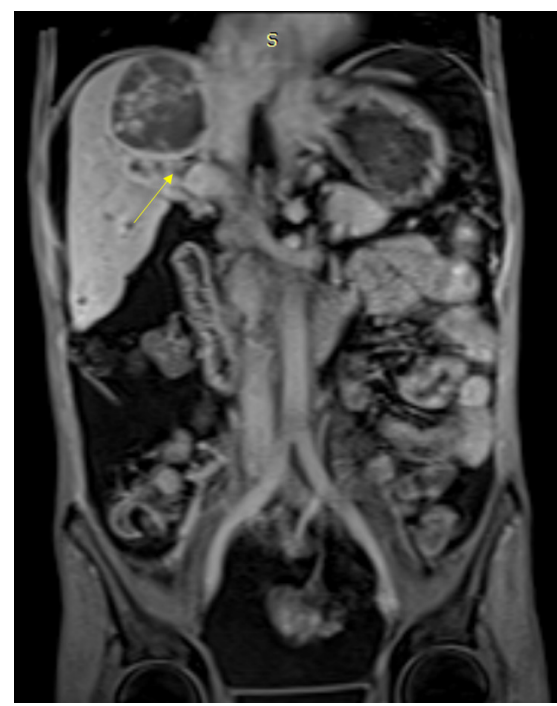

Figure 2 Description: Late-stage post-contrast coronal magnetic resonance imaging in TIW. Tumor thrombus that enhances in the venous phase and expands the inferior vena cava (arrow).

Origin: Department of Radiology, Clínica de la Costa, Barranquilla, Colombia, 2019.

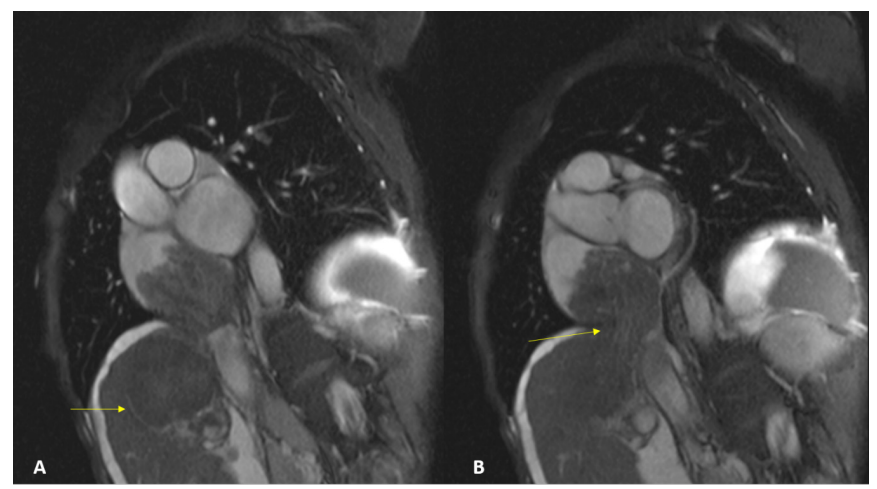

Figure 3 Description: Sagittal magnetic resonance imaging in T2W (A) and TIW (B). Hepatocarcinoma extends through the inferior vena cava to the right atrium, invading more than $50 \%$ and in contact with the atrioventricular leaflets. Origin: Department of Radiology, Clínica de la Costa, Barranquilla, Colombia, 2019.

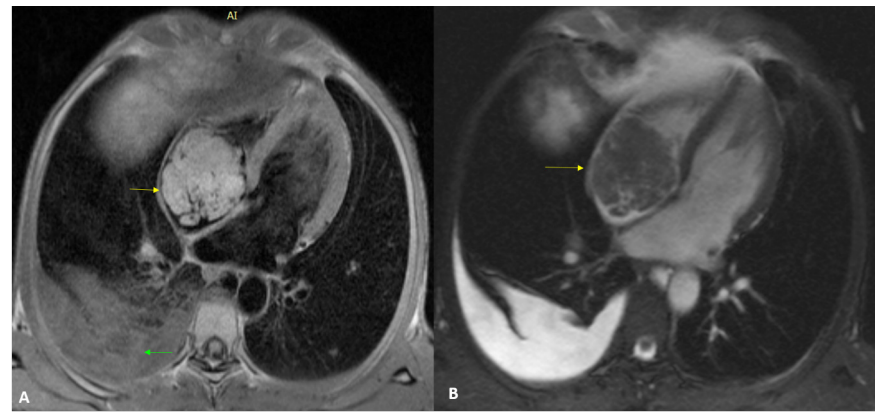

Figure 4 Description: Axial magnetic resonance TIW Fatsat $(A)$ and T2W cinema post-contrast. Extensive atrial involvement due to metastatic tumor invasion. Right pleural effusion (green arrow) is also observed. Origin: Department of Radiology, Clínica de la Costa, Barranquilla, Colombia, 2019.

\section{Discussion}

Hepatocarcinoma with diffuse subtype is a rare diagnosis with few reviews in the literature, corresponding to approximately $7-13 \%$ of cases of hepatocellular carcinoma. ${ }^{4}$ As for tumor markers, some studies have shown that alpha-fetal protein (AFP) is a highly specific parameter, with high protein values $(>400 \mathrm{ng} / \mathrm{ml}$, check your laboratory reference values), with a low sensitivity of $54 \%$. This indicates that we should pay special attention to elevated AFP values, while low values do not exclude the diagnosis of hepatocellular carcinoma. ${ }^{6}$

Diffuse hepatocellular carcinoma has an infiltrating behavior, therefore, in the image, an alteration in the intensity of the homogeneous or heterogeneous variable signal is observed. Magnetic resonance imaging (MRI) shows: ${ }^{7,8}$

a. Predominantly hypointense lesion in $\mathrm{T} 1$ and $\mathrm{T} 2$ varies from hypo to isointense.

b. After administration of the contrast medium, a "miliar" pattern is identified

c. Dynamic post-contrast images with heterogeneous areas of enhancement in early phases and "washing" in later phases.

d. Restriction on dissemination.

e. There is a high prevalence of portal vein thrombosis in patients with HCC, with unique characteristics in the diffuse subtype, given by an invasion pattern with marked distension of the vein which as it infiltrates the adjacent vessels acquires an image of molding of these. ${ }^{6}$

f. In the portal phase, the portal thrombus is observed as a filling defect.

Differential imaging diagnoses include cholangiocarcinoma, atrial myxoma, and cardiac angiosarcoma. Patients with diffuse hepatocellular carcinoma have a poor prognosis in relation to the late stage of presentation and diagnosis. This subtype is a contraindication to resection or transplantation, with transcatheter chemo-embolization as the only therapeutic option for these patients. ${ }^{8}$

\section{Conclusion}

It is essential to recognize the MRI findings of diffuse hepatocarcinoma, in order to intervene in a timely manner.

\section{Funding}

There was no funding for this report.

\section{Acknowledgments}

None.

\section{Conflicts of interest}

Author declares there are no conflicts of interest.

\section{References}

1. Ghouri YA, Mian I, Rowe JH. Review of hepatocellular carcinoma: Epidemiology, etiology, and carcinogenesis. J Carcinog. 2017;16:1.

2. Lafaro KJ, Demirjian AN, Pawlik TM. Epidemiology of hepatocellular carcinoma. Surg Oncol Clin N Am. 2015;24(1):1-17.

3. Franceschi S, Raza SA. Epidemiology and prevention of hepatocellular carcinoma. Cancer Lett. 2009;286(1):5-8

4. Trevisani F, Caraceni P, Bernardi M, et al. Gross pathologic types of hepatocellular carcinoma in Italian patients. Relationship with demographic, environmental, and clinical factors. Cancer. 1993;72(5):1557-1563. 
5. Kanematsu M, Semelka RC, Leonardou P, et al. Hepatocellular carcinoma of diffuse type: MR imaging findings and clinical manifestations. J Magn Reson Imaging. 2003;18(2):189-195.

6. Demirjian A, Peng P, Geschwind JFH, et al. Infiltrating hepatocellular carcinoma: Seeing the tree through the forest. J Gastrointest Surg. 2011;15(11):2089-2097.
7. Reynolds AR, Furlan A, Fetzer DT, et al. Infiltrative hepatocellular carcinoma: what radiologists need to know. Radiographics. 2015;35(2):371-386.

8. Catalano O, Choy G, Zhu A, et al. Differentiation of malignant thrombus from bland thrombus of the portal vein in patients with hepatocellular carcinoma: application of diffusion-weighted MR imaging. Radiology. 2010;254(1):154-162. 